\title{
Study and Application of Digital Image Reconstruction
}

\author{
Yan Wenzhong* and Bai Lei
}

Department of Computer, North China Institute of Science and Technology, Sanhe, Hebei, 065201, P.R. China

\begin{abstract}
Digital image reconstruction is a sound method to reveal the underlying images hidden in fuzzy and noisy data. The main defiance of this method is its sensitivity to measure noise in the input data, which will result in large artifacts in the reconstructed image. So it has become very important that finding a robust method to remove the noise and reconstruct the image. For the purpose of acquiring a gray image without loosing information from it, a method of removing noise from a gray image and reconstructing this image was proposed in this paper. The proposed method mainly used the opening-by-reconstruction. In order to test the efficacy of this method, it is used to isolate the text out of the image of calculator keys. The experimental results showed that the method is efficient.
\end{abstract}

Keywords: Morphological operations, opening-by-reconstruction, reconstruction.

\section{INTRODUCTION}

Digital images may be affected by a lot of types of noise. The noise can be introduced into one image by several ways, which depends on how this image is created. For instance: An image is created by scanning from a picture made on film, so the film grain may become one source of noise. Meanwhile the noise can also result from the scathe of the film, and even the scanner itself can be a source of noise. Another image may be obtained directly in the digital format, so the noise can be introduced by the mechanism for collecting the data. And the noise can also be introduced by electronic transmission of image data [1-4].

Rather than a single image and structuring element, reconstruction is a morphological transformation, which involve two images and a structuring element. The transformation starts from one image (called the marker). And this transformation is restrained by the other image (called the mask). The connectivity is defined by the structuring element. In this paper, 8-connectivity is employed. And in the following discussion, $\mathrm{B}$ is a $3 \times 3$ matrix of 1 s, whose center is at coordinates $(2,2)$.

Let's suppose that $g$ is the marker and $f$ is the mask. It is denoted as $R_{f}(g)$ that the reconstruction of $f$ from $g$, which is defined by the following repetitious process:

1. Initialize $h_{l}$ to be the marker image $g$.

2. Create the structuring element $: B=$ ones(3).

3. Repeat:

$h_{k+1}=\left(h_{k} \oplus B\right) \cap f$

until $\mathrm{h}_{\mathrm{k}+1}=\mathrm{h}_{\mathrm{k}}$.

Marker $\mathrm{g}$ must be a subset of $\mathrm{f}$; that is $\mathrm{g} \cap \mathrm{f}$

This paper is focused on the digital image reconstruction.

First, the theory of mathematical morphology and reconstruction is studied. Then a method for removing noise from a gray image and reconstructing this image is proposed. Finally, an experiment is conducted to test the efficacy of the proposed method. The description of the method in detail, the experimental results and the analysis of the problems occurred in the experiment, are all presented here.

\section{BASIC THEORY}

\subsection{Mathematical Morphology}

Mathematical Morphology is used to extract image components which are good for description and representation. This technique was firstly developed by Matheron and Serra at the Ecole des Mines in Paris. The Mathematical Morphology is a set-theoretic method used for image analysis which provides a quantitative description of geometrical structures. Borders of objects, their skeletons, and their convex hulls can be provided by morphology. Morphology can also be used for a lot of pre- and post-processing methods, such as edge pruning and thinning.

In general, most morphological operations are based on easy shrinking and expanding operations. Although morphology can be used on grey level images, its primary application occurs in binary images. Meanwhile, it can also be used on range images.

There are two basic morphological set transformations, erosion and dilation. The interaction is contained in these transformations, which is between an image $\mathrm{A}$ and a structuring set B. A is the object of interest and B is the structuring element.

Although the structuring element B can be any shape, the shape is usually a circular disc in the plane. Both the structuring element sets and the image can be defined in 1-D, 2- 
D, 3-D or higher dimensions, instead of being defined restrictedly in the $2 \mathrm{D}$ plane.

Let $A$ and $B$ be subsets of $Z^{2}$. Then the translation of $A$ by $\mathrm{x}$ is denoted as $A_{x}$, which is defined as

$$
A_{x}=\{c: c=a+x, \text { for } a \in A\} .
$$

The reflection of B is denoted as $\hat{B}$, which is defined as

$\hat{B}=\{x: x=-b$, for $b \in B\}$.

Ac denotes the complement of A and A - B. denotes the difference of two sets $\mathrm{A}$ and $\mathrm{B}$.

1) Dilation

Using the structuring element B to dilate the object A is defined as

$$
A \oplus B=\left\{x: \hat{B}_{x} \cap A \neq \emptyset\right\} .
$$

The result of this processing is a new set. This new set is made up of all points which are created by obtaining the reflection of $\mathrm{B}$ about its origin and then using $\mathrm{x}$ to shift this reflection.

Think of an example where $\mathrm{A}$ is a rectangle and $\mathrm{B}$ is a disc centered on the origin. Because B is symmetric,

$$
\hat{B}=B
$$

This definition turns into very intuitive when the structuring element B is considered as a convolution mask.

\section{2) Erosion}

Erosion of the object A by a structuring element B is defined as

$$
A \ominus B=\left\{x: B_{x} \subseteq A\right\} .
$$

Dilation and Erosion can be used in various ways, both in series and parallel, which can give other transformations including thinning, skeletonisation, thickening and many others.

Opening and closing are two very important transformations. The erosion shrinks one image object and dilation expands it. Generally speaking, opening always smooths the contour in an image, eliminating thin protrusions and breaking narrow isthmuses. Closing always narrows smooth sections of contours, eliminating small holes, filling gaps in contours, long thin gulfs and fusing narrow breaks.

The opening of $\mathrm{A}$ by $\mathrm{B}$ is denoted as $A \circ B$. It is acquired by the erosion by $\mathrm{B}$ and then the dilation by $\mathrm{B}$, it is defined as

$$
A \circ B=(A \ominus B) \oplus B .
$$

Generally speaking opening is like rounding from the inside. The opening of $\mathrm{A}$ by $\mathrm{B}$ is acquired by taking the union of all translates of $B$ which fit inside $A$ and removing the parts of A which are smaller than B. Thus

$$
A \circ B=\bigcup\left\{B_{x}: B_{x} \subseteq A\right\} \text {. }
$$

The closing of A by $\mathrm{B}$ is denoted as $A \circ B$, which is the dual operation of opening. It is acquired by the dilation by $\mathrm{B}$ and then the erosion by $\mathrm{B}$, it is defined as

$$
A \bullet B=(A \oplus B) \ominus B \text {. }
$$

Closing is like smoothing from the outside. After closing, narrow valleys are closed and holes are filled in.

Opening and closing are dual operations just like dilation and erosion. That is

$$
(A \bullet B)^{c}=\left(A^{c} \circ B^{c}\right) \text {. }
$$

One image can be indicated by a series of pixels. Two images are used by the morphologic operators: one is the original data that will be analyzed, the other is a structuring element which is analogous to the kernel of a convolution operation. The shape of each structuring element is thought to be one parameter of the operation.

\subsection{Basic Theory of Resconstruction}

In the morphological reconstruction, there are two images, the mask image and the marker image. So the morphological reconstruction can be described as that the marker image is dilated repeatedly until its contour fits under the mask image. During morphological reconstruction, the peaks in the marker image dilate or expand, Fig. (1) illustrates the reconstruction in 1-D. Every continuous dilation is limited to lie beneath the mask. This processing will not stop unless the further dilation stops to change the image. Then the final dilation becomes the reconstructed image. The following figure shows the continuous dilations of the marker.

Morphological dilation is the base of morphological reconstruction. But the morphological reconstruction has the following sole properties, which are different from the morphological dilation:

The morphological dilation is based on one image and a structuring element. But morphological reconstruction is based on two images, the marker and the mask.

The morphological dilation is based on a structuring element. But morphological reconstruction is based on the concept of connectivity.

This processing will repeat until stability; that is to say, the image doesn't change any longer.

Another useful gray-scale reconstruction technique is opening-by-reconstruction. Firstly, as in standard morphological opening, an image is eroded. Secondly, in a reconstruction, this eroded image is used as the marker image. Meanwhile, the original image is used as the mask image.

Reconstruction can be used to clean up image further by applying to a technique called closing-by-reconstruction. The implementation steps of closing-by-reconstruction are as follows:

(1). Complementing an image.

(2). Computing its opening-by-reconstruction.

(3). Complementing the result. 


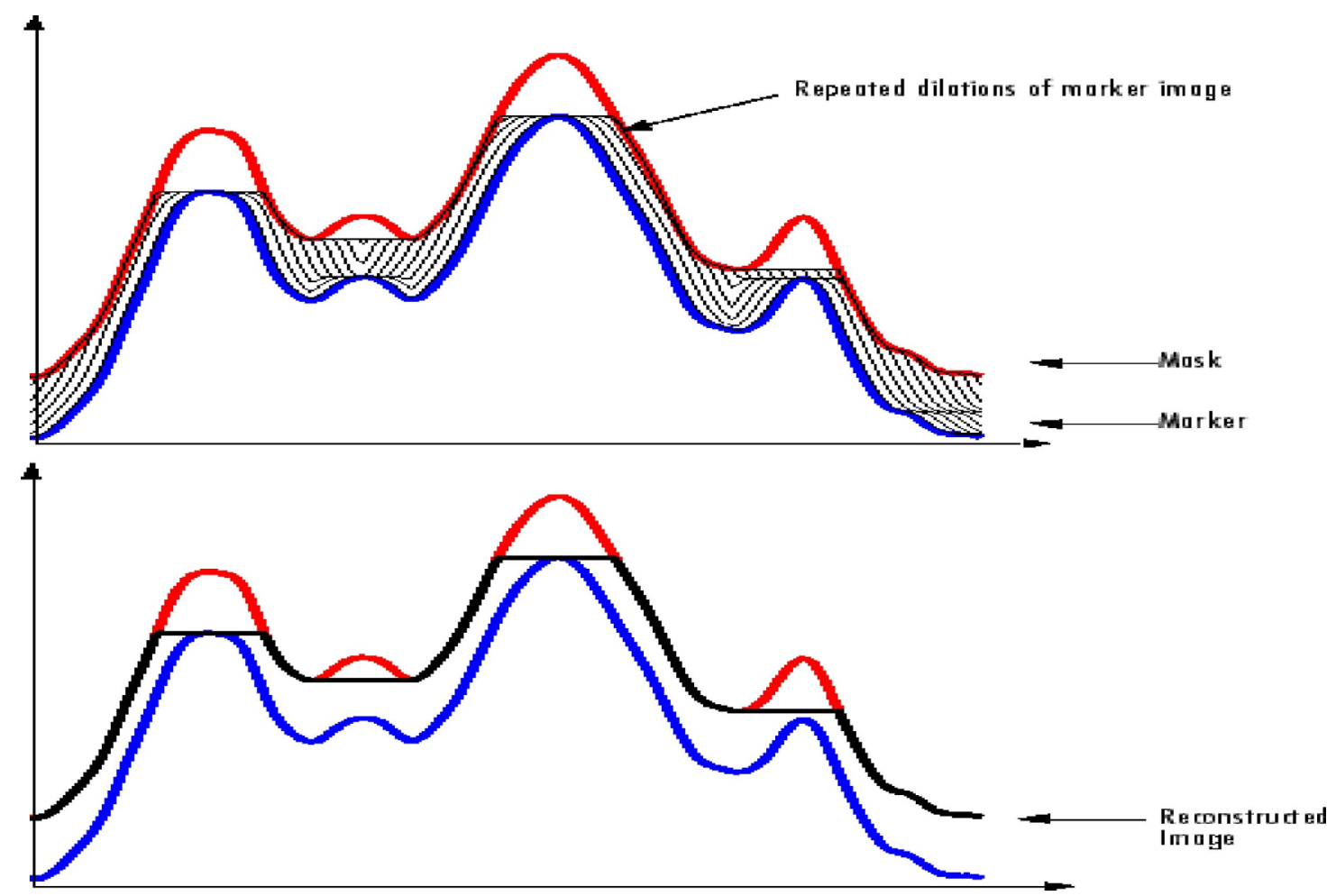

Fig. (1). Processing of morphological reconstruction.

\section{METHOD AND EXPERIMENTAL RESULTS}

In this paper, the gray-scale reconstruction is used in several steps. In order to test the efficacy of this method, an experiment is conducted to isolate the text out of the image of calculator keys. The description of the method in detail and the experimental results are presented as follow.

The objective is to isolate the text out of the image of calculator keys shown in Fig. (2).

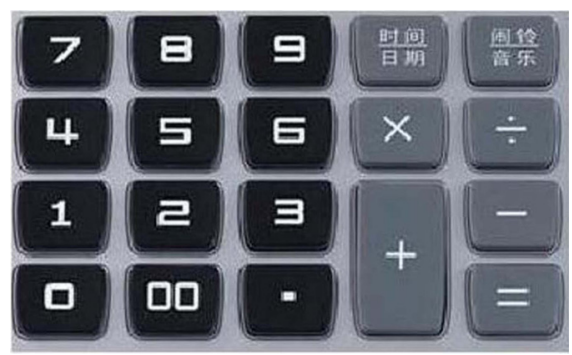

Fig. (2). Original image.

The first step is to suppress the horizontal reflections along the top of each key. To accomplish this, we take advantage of the fact that these reflections are wider than any single text character in the image. We perform opening-byreconstruction using a structuring element that is a long horizontal line.

The result of opening-by-reconstruction is shown in Fig. (3). For comparison, Fig. (4) shows the result of standard opening. Opening-by-reconstruction did a better job of extraction the background between horizontally adjacent keys. Subtracting the opening-by-reconstruction from the original image is called tophat-by-reconstruction, and is shown in Fig. (5).

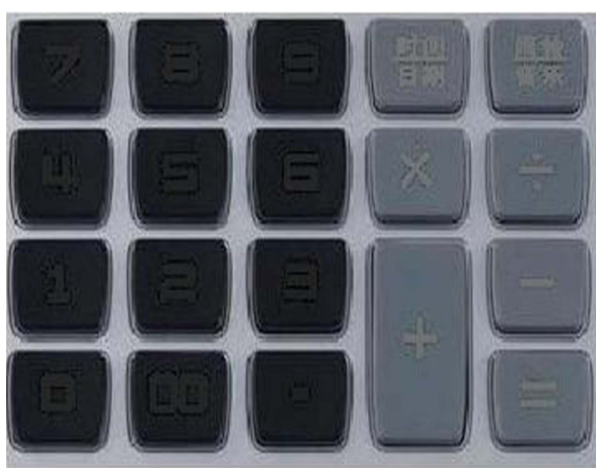

Fig. (3). Result of opening-by-reconstruction.

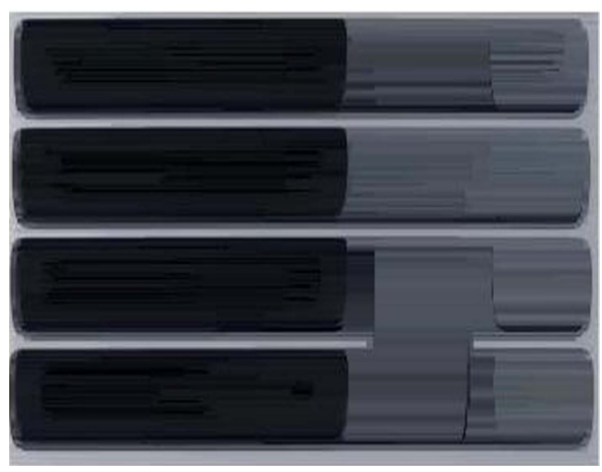

Fig. (4). Result of standard opening.

Fig. (6) shows the result of standard top-hat computation.

Next, the vertical reflections on the right edges of the keys were suppressed in Fig. (5). This is done by performing opening-by-reconstruction with a small horizontal line. 


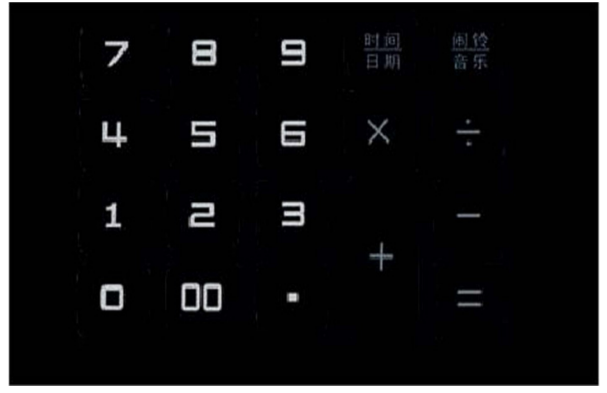

Fig. (5). Result of Tophat-by-reconstruction.

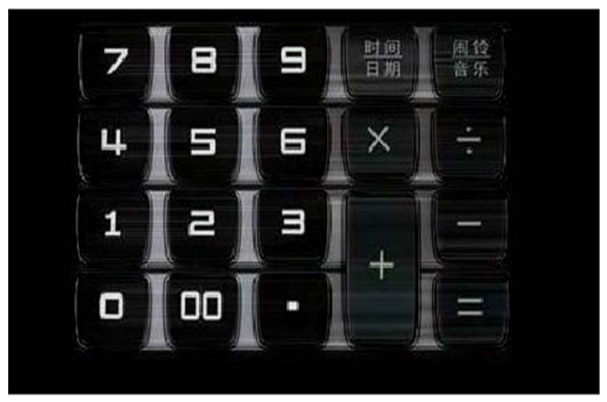

Fig. (6). Result of standard top-hat computation.

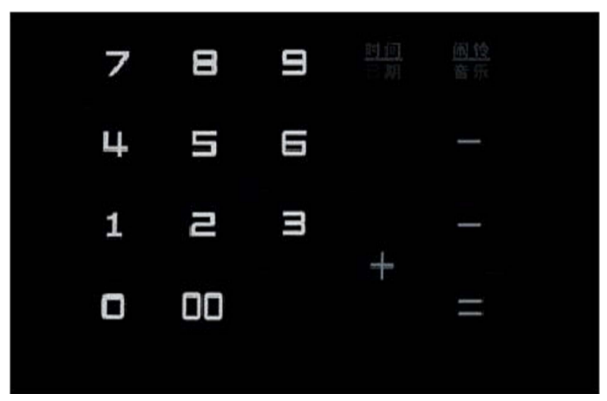

Fig. (7). Result of opening-by-reconstruction of Fig. (5) by using a horizontal line.

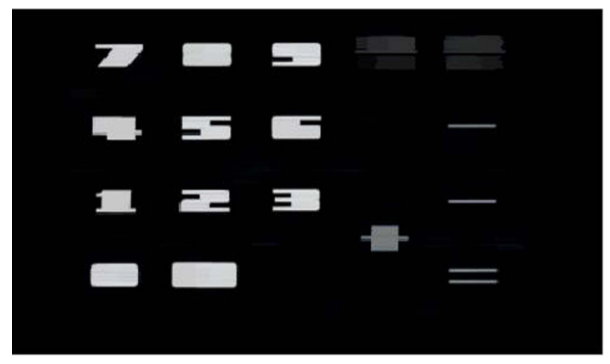

Fig. (8). Result of dilation of Fig. (7) by using a horizontal line.

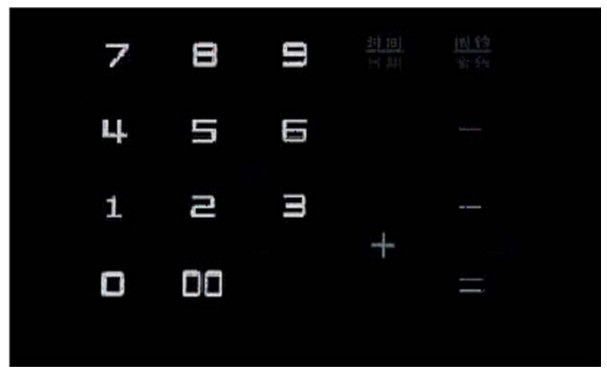

Fig. (9). Final reconstruction result.

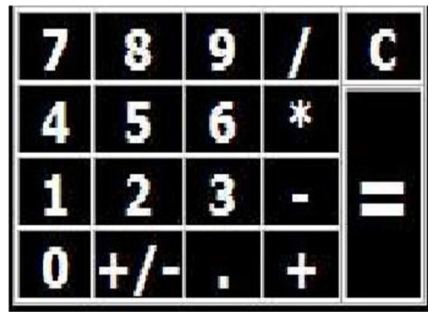

Fig. (10). Original image.

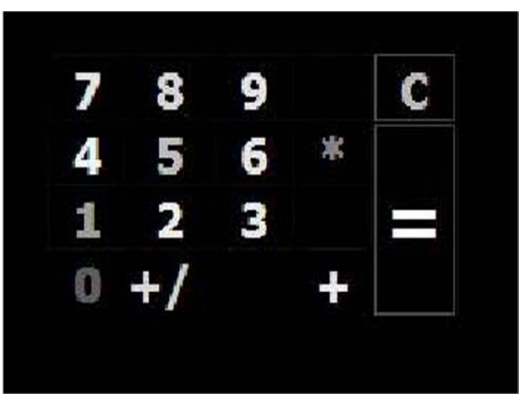

Fig. (11). Result of reconstruction of Fig. (10).

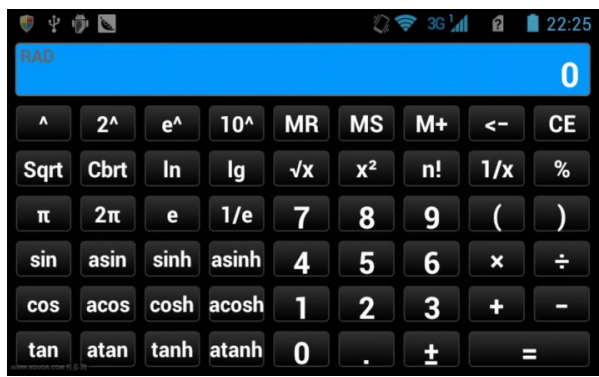

Fig. (12). Original image.

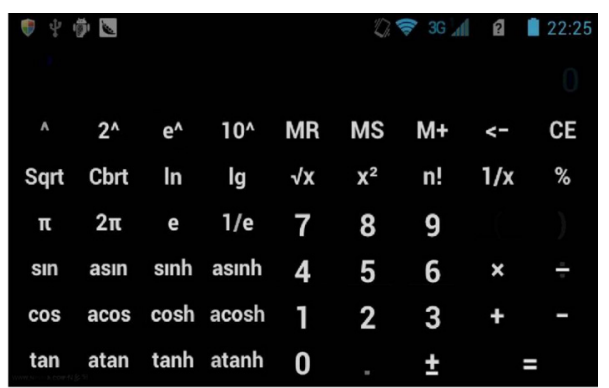

Fig. (13). Result of Reconstruction of Fig. (12).

In the result Fig. (7), it can be seem that the vertical reflections are gone, so are the thin-vertical-stroke characters, such as the point symbol. We take advantage of the fact that the characters that have been suppressed in error are very close to other characters still present by first performing a dilation Fig. (8).

Followed by a final reconstruction with Fig. (5) as the mask and min (Fig. 8, Fig. 5) as the marker, Fig. (9) shows the final result. Note that the shading and reflections on the background and keys were removed successfully.

In order to test the efficacy of this method, some other calculator images are processed by the method. Figs. (10-13) shows the experiment results. Figs. (10 and 12) are the original image, Figs. (11 and 13) are the results of the reconstruction. 


\section{CONCLUSION}

In this paper, the basic theories of mathematical morphology and digital image reconstruction are studied. Then the gray-scale reconstruction is used to isolate the text out of the image of calculator keys. The experimental result shows that the proposed method is efficient. But there are still some problems. For example, the thin-vertical-stroke characters in the image are gone, such as the point symbol. These problems need further research to solve.

\section{CONFLICT OF INTEREST}

The authors confirm that this article content has no conflict of interest.

\section{ACKNOWLEDGEMENTS}

This work was financially supported by the National Natural Science Foundation of China (No.61163050, No.
61472137), NCST Program (No. 3142013098, No.3142014125), the Higher school science research project of Hebei Province (Z2014038) and the Fundamental Research Funds for the Central Universities (2011A010 and 3142014007).

\section{REFERENCES}

[1] R. M. Rangayyan, and A. Das, "Filtering multiplicative noise in images using adaptive region based statistics", Journal of Electronic Imaging, vol. 7, no. 1, pp. 222-230, 1998.

[2] B. Aiazzi, S. Baronti, and L. Alparone, "Multi resolution adaptive filtering of signal-dependent noise based on a generalized laplacian pyramid", In: Proceedings of International Conference on Image Processing, Santa Barbara, CA, vol. 1, pp. 381-384, 1997.

[3] K. Jostschulte, A. Amer, M. Schu, and H. Schroder, "Perception adaptive temporal tv-noise reduction using contour preserving prefilter techniques", Consumer, vol. 44, no. 3, pp. 1091-1096, 1998.

[4] Y. Boutalis, S. Kollias, and G. Carayannis, "A fast adaptive approach to the restoration of images degraded by noise", Signal Processing, vol. 19, no. 2, pp. 151-162, 1990.

(C) Wenzhong and Lei; Licensee Bentham Open.

This is an open access article licensed under the terms of the (https://creativecommons.org/licenses/by/4.0/legalcode), which permits unrestricted, noncommercial use, distribution and reproduction in any medium, provided the work is properly cited. 